MATHEMATICS OF COMPUTATION

Volume 76, Number 257, January 2007, Pages 233-249

S 0025-5718(06)01907-7

Article electronically published on October 11, 2006

\title{
BIORTHOGONAL BASES WITH LOCAL SUPPORT AND APPROXIMATION PROPERTIES
}

\author{
BISHNU P. LAMICHHANE AND BARBARA I. WOHLMUTH
}

\begin{abstract}
We construct locally supported basis functions which are biorthogonal to conforming nodal finite element basis functions of degree $p$ in one dimension. In contrast to earlier approaches, these basis functions have the same support as the nodal finite element basis functions and reproduce the conforming finite element space of degree $p-1$. Working with Gauß-Lobatto nodes, we find an interesting connection between biorthogonality and quadrature formulas. One important application of these newly constructed biorthogonal basis functions are two-dimensional mortar finite elements. The weak continuity condition of the constrained mortar space is realized in terms of our new dual bases. As a result, local static condensation can be applied which is very attractive from the numerical point of view. Numerical results are presented for cubic mortar finite elements.
\end{abstract}

\section{INTRODUCTION}

We construct basis functions which are biorthogonal to conforming nodal onedimensional finite element basis functions of degree $p$ with respect to the $L^{2}$-norm. By definition our newly constructed basis functions have the same support as the conforming nodal basis functions. Using Gauß-Lobatto nodes to define the conforming nodal basis functions of degree $p$, our biorthogonal basis functions span a nonconforming finite element space which includes the conforming finite element space of degree $p-1, p>1$. Using this type of nonconforming space as Lagrange multiplier space for two-dimensional mortar finite elements results in an optimal discretization scheme. We note that the Lagrange multiplier space has to be modified in a suitable way in the neighborhood of the crosspoints to satisfy a uniform inf-sup condition. The natural norm in the mortar setting for the Lagrange multiplier is the broken $H^{-1 / 2}$-norm on the interior interfaces. Thus to obtain an order $h^{p}$ a priori result, it is sufficient to reproduce polynomials of degree $p-1$ in the Lagrange multiplier space. Due to the biorthogonality, the arising mass matrices on the slave side of the mortar formulation are diagonal, and a local static condensation can be carried out. Physically, this corresponds to a lumping of the mass matrix, and the locality reflects the fact that a local perturbation influences the solution only in a small neighborhood. Therefore, these dual Lagrange multiplier spaces are

Received by the editor April 7, 2005 and, in revised form, October 20, 2005.

2000 Mathematics Subject Classification. Primary 65N30, 65N55, 65D32.

Key words and phrases. Biorthogonal basis, domain decomposition, Lagrange multipliers, reproduction property.

This work was supported in part by the Deutsche Forschungsgemeinschaft, SFB 404, C12. 
very attractive from computational and physical points of view. Such a biorthogonal basis was introduced in Whoh0] for lowest order mortar finite elements, i.e., for degree $p=1$, and was called the dual Lagrange multiplier base. Recently low order mortar finite elements with a dual Lagrange multiplier has become an active area of research; see [KLPV01, Mar05, Ben04, HT04a, HT04b. The idea has also been extended to higher order elements; see [LW02, LSW05, OW01]. We refer to DS99, Ste03 for related work in biorthogonal multiresolution analysis. However, the construction of biorthogonal bases is not straightforward, and preserving the locality of the support is not trivial. In OW01, one-dimensional higher order dual finite element bases are considered. The drawback of this technique is that the support has to be extended, resulting in a more complex density pattern in the mass matrices. Here, we focus on the construction of biorthogonal basis functions having the same support as the nodal basis functions.

The rest of the paper is organized as follows. In the next section, introducing some notation and the concept of biorthogonality, we construct our dual basis starting from a given set of nodal basis functions on a reference element. Using Gauß-Lobatto nodes, the dual basis reproduces the finite element space of degree $p-1$. In Section 3, we consider the application to mortar finite elements. We work out the required modification at the endpoints of the interfaces. Finally, numerical results are presented in Section 4.

\section{Dual basis in one Dimension}

Before giving the explicit construction, we consider an abstract framework. Let $V$ and $W$ be finite-dimensional subspaces of a Hilbert space $H$ with inner product $(\cdot, \cdot)$. We assume that $\operatorname{dim} V=\operatorname{dim} W$ and associate the space $V$ with the basis set $\boldsymbol{\Phi}:=\left\{\phi_{1}, \ldots, \phi_{n}\right\}$ and $W$ with the basis set $\boldsymbol{\Lambda}:=\left\{\lambda_{1}, \ldots, \lambda_{n}\right\}$. To make a consistent matrix notation, all basis sets are also thought of as column vectors. We denote by $G_{\boldsymbol{\Phi}, \boldsymbol{\Lambda}}=\left(\boldsymbol{\Phi}, \boldsymbol{\Lambda}^{T}\right)$ the Gram matrix associated with the two finite systems $\boldsymbol{\Phi}$ and $\boldsymbol{\Lambda}$ based on the inner product $(\cdot, \cdot)$, i.e., $G_{\boldsymbol{\Phi}, \boldsymbol{\Lambda}}$ is an $n \times n$ matrix with $\left(G_{\boldsymbol{\Phi}, \boldsymbol{\Lambda}}\right)_{i j}=\left(\phi_{i}, \lambda_{j}\right)$.

Definition 2.1. The set $\Lambda$ will be called a dual basis with respect to $\Phi$ if and only if $G_{\boldsymbol{\Phi}, \boldsymbol{\Lambda}}=D_{n}$, where $D_{n}$ is a nonsingular $n \times n$ diagonal matrix.

Let $\mathcal{S}_{p}:=\left\{-1=: x_{1}^{p}<x_{2}^{p}<\cdots<x_{p+1}^{p}:=1\right\}$ be a set of distinct points in the reference interval $\bar{I}:=[-1,1]$, and let $\hat{\boldsymbol{\Phi}}_{p}:=\left\{\phi_{1}^{p}, \phi_{2}^{p}, \ldots, \phi_{p+1}^{p}\right\}, \phi_{i}^{p} \in \mathcal{P}_{p}(I)$, $1 \leq i \leq p+1$, be the associated set of nodal finite element basis functions, i.e., $\phi_{i}^{p}\left(x_{j}^{p}\right)=\delta_{i j}$, where $\delta_{i j}$ is the Kronecker symbol. Here, $\mathcal{P}_{p}(I)$ denotes the $(p+1)$ dimensional space of polynomials of degree less than or equal to $p$. From now on, we simply call this basis a finite element basis of degree $p$ based on $\mathcal{S}_{p}$. Associated with $\hat{\mathbf{\Phi}}_{p}$ is the dual basis $\hat{\boldsymbol{\Lambda}}_{p}:=\left\{\lambda_{1}^{p}, \lambda_{2}^{p}, \ldots, \lambda_{p+1}^{p}\right\}$, where the $\lambda_{i}^{p} \in \mathcal{P}_{p}(I), 1 \leq i \leq p+1$, are uniquely defined by

$$
\int_{I} \lambda_{i}^{p}(\hat{s}) \phi_{j}^{p}(\hat{s}) d \hat{s}=d_{i}^{p} \delta_{i j}, \quad d_{i}^{p} \neq 0 .
$$

We set $d_{i}^{p}:=\int_{I} \phi_{i}^{p}(\hat{s}) d \hat{s}$ if the integral is nonzero, and $d_{i}^{p}:=\int_{I} d \hat{s}$ otherwise. It is trivial to see that the spaces spanned by $\hat{\boldsymbol{\Phi}}_{p}$ and $\hat{\boldsymbol{\Lambda}}_{p}$ are the same. Moreover for $q \leq p$ there exists a unique $(q+1) \times(p+1)$ matrix $N^{q ; p}$ such that

$$
\hat{\boldsymbol{\Phi}}_{q}=N^{q ; p} \hat{\boldsymbol{\Lambda}}_{p} .
$$


To define our global spaces, we introduce a locally quasi-uniform finite element mesh $\mathcal{I}_{h}$ which is formed by partitioning a segment $\gamma$ into $K$ subintervals (elements), $I_{k}$, $1 \leq k \leq K$, with $\bar{\gamma}=\bigcup_{k=1}^{K} \bar{I}_{k}$. The ordering of the $I_{k}$ is done in a lexicographical way from the left to the right. Associated with each element $I_{k}$ is a unique affine mapping $F_{k}: \bar{I} \longrightarrow \bar{I}_{k}$ which maps -1 to the left and 1 to the right endpoint of $I_{k}$. Due to the ordering, we find that $F_{k}\left(x_{1}^{p}\right)=F_{k-1}\left(x_{p+1}^{p}\right), 2 \leq k \leq K$. We transform the basis functions $\phi_{i}^{p}$ and $\lambda_{i}^{p}$ on $I$ in the standard way, and set $\boldsymbol{\Phi}_{p}^{k}:=$ $\left\{\phi_{k ; 1}^{p}, \phi_{k ; 2}^{p}, \ldots, \phi_{k ; p+1}^{p}\right\}$ and $\boldsymbol{\Lambda}_{p}^{k}:=\left\{\lambda_{k ; 1}^{p}, \lambda_{k ; 2}^{p}, \ldots, \lambda_{k ; p+1}^{p}\right\}$, where $\phi_{k ; i}^{p}:=\phi_{i}^{p} \circ F_{k}^{-1}$ and $\lambda_{k, i}^{p}:=\lambda_{i}^{p} \circ F_{k}^{-1}$. Then it is easy to see that the following biorthogonality relation holds:

$$
\int_{I_{k}} \lambda_{k ; i}^{p}(s) \phi_{k ; j}^{p}(s) d s=\frac{\left|I_{k}\right|}{2} d_{i}^{p} \delta_{i j},
$$

and we have $\boldsymbol{\Phi}_{q}^{k}=N^{q ; p} \boldsymbol{\Lambda}_{p}^{k}$ with the same $N^{q ; p}$ as in (2.2). Associated with each node $x_{j} \in \bar{\gamma}, 1 \leq j \leq p K+1, x_{1}:=F_{1}(-1), x_{p(k-1)+j}:=F_{k}\left(x_{j}^{p}\right), 1 \leq k \leq K$, $2 \leq j \leq p+1$, is exactly one basis function in $\boldsymbol{\Phi}_{p}$ and $\boldsymbol{\Lambda}_{p}$. We define

$$
\phi_{j}^{p}:= \begin{cases}\phi_{k ; i}^{p}, & j=p(k-1)+i, 2 \leq i \leq p, 1 \leq k \leq K, \\ \phi_{1 ; 1}^{p}, & j=1, \\ \phi_{K ; p+1}^{p}, & j=p K+1, \\ \phi_{k ; p+1}^{p}+\phi_{k+1 ; 1}^{p}, & j=p k+1,1 \leq k<K,\end{cases}
$$

where all basis functions $\phi_{k ; j}^{p}$ are extended by zero outside of $\bar{I}_{k}$, and the addition of two basis functions " $\phi_{k ; p+1}^{p}+\phi_{k+1 ; 1}^{p}$ " has to be interpreted such that $\left.\phi_{j}^{p}\right|_{\bar{I}_{k}}=\phi_{k ; p+1}^{p}$ and $\left.\phi_{j}^{p}\right|_{\bar{I}_{k+1}}=\phi_{k+1 ; 1}^{p}$ for $j=p k+1,1 \leq k<K$. We note that this is well defined because $\phi_{k ; p+1}^{p}\left(x_{p k+1}\right)=\phi_{k+1 ; 1}^{p}\left(x_{p k+1}\right)$. It is well known that the basis $\boldsymbol{\Phi}_{p}:=\left\{\phi_{1}^{p}, \ldots, \phi_{p K+1}^{p}\right\}$ spans the conforming finite element space $V_{h}^{p}$ of degree $p$ associated with the one-dimensional mesh $\mathcal{I}_{h}$. The elements of the dual basis $\boldsymbol{\Lambda}_{p}:=$ $\left\{\lambda_{1}^{p}, \ldots, \lambda_{p K+1}^{p}\right\}$ of $\boldsymbol{\Phi}_{p}$ are constructed from the local basis functions similarly as those of $\boldsymbol{\Phi}_{p}$. We note that the global basis functions $\lambda_{j}^{p}$ are in general discontinuous at the endpoints of the elements. As a result, the space $W_{h}^{p}$ spanned by the basis functions in $\boldsymbol{\Lambda}_{p}$ is a nonconforming finite element space. Moreover, we will find that the finite element space of degree less than or equal to $p$ on $\gamma$ will not be included in $W_{h}^{p}$. A first result in this direction is provided by the following lemma.

Lemma 2.2. $V_{h}^{q} \subset W_{h}^{p}$ if and only if

$$
\begin{array}{ll}
n_{1,1}^{q ; p}= & n_{q+1, p+1}^{q ; p} \quad \text { and } \quad n_{q+1,1}^{q ; p}=n_{1, p+1}^{q ; p}=0, \\
n_{i, p}^{q ; p}= & n_{i, p+1}^{q ; p}=0 \quad \text { for all } \quad 2 \leq i \leq q .
\end{array}
$$

where $n_{i, j}^{q ; p}$ is the $(i, j)$-th entry of the matrix $N^{q ; p}$.

Proof. The dimension of $V_{h}^{q}$ is given by $q K+1$. In a first step, we consider the basis functions $\phi_{j}^{q}, j=q(k-1)+i, 2 \leq i \leq q, 1 \leq k \leq K$, in more detail. Using the definition of the global basis functions, we find

$\phi_{j}^{q}=\phi_{k ; i}^{q}=\left(N^{q ; p} \boldsymbol{\Lambda}_{p}^{k}\right)_{i}=\sum_{l=1}^{p+1} n_{i, l}^{q ; p} \lambda_{k ; l}^{p}=n_{i, 1}^{q ; p} \lambda_{k ; 1}^{p}+\sum_{l=2}^{p} n_{i, l}^{q ; p} \lambda_{p(k-1)+l}^{p}+n_{i, p+1}^{q ; p} \lambda_{k ; p+1}^{p}$.

As a result, we obtain that $\phi_{j}^{q} \in W_{h}^{p}, j=q(k-1)+i, 2 \leq i \leq q, 1 \leq k \leq K$, if and only if $n_{i, 1}^{q ; p}=n_{i, p+1}^{q ; p}=0,2 \leq i \leq q$. Secondly, we consider the basis functions of $V_{h}^{q}$ which are associated with an endpoint of one element. We do not work out the 
details for $\phi_{1}^{q}$ and $\phi_{q K+1}^{q}$ but concentrate on $\phi_{q k+1}^{q}, 1 \leq k<K$. Each $\phi_{q k+1}^{q}$ can be written as an element-wise sum of two local basis functions

$$
\begin{aligned}
\phi_{q k+1}^{q}= & \phi_{k ; q+1}^{q}+\phi_{k+1 ; 1}^{q}=\left(N^{q ; p} \boldsymbol{\Lambda}_{p}^{k}\right)_{q+1}+\left(N^{q ; p} \boldsymbol{\Lambda}_{p}^{k+1}\right)_{1} \\
= & \sum_{l=1}^{p+1}\left(n_{q+1, l}^{q ; p} \lambda_{k ; l}^{p}+n_{1, l}^{q ; p} \lambda_{k+1 ; l}^{p}\right) \\
= & n_{q+1,1}^{q ; p} \lambda_{k ; 1}^{p}+\left(n_{q+1, p+1}^{q ; p}-n_{1,1}^{q ; p}\right) \lambda_{k ; p+1}^{p}+n_{1, p+1}^{q ; p} \lambda_{k+1 ; p+1}^{p} \\
& +n_{1,1}^{q ; p} \lambda_{p k+1}^{p}+\sum_{l=2}^{p}\left(n_{q+1, l}^{q ; p} \lambda_{p(k-1)+l}^{p}+n_{1, l}^{q ; p} \lambda_{p k+l}^{p}\right) .
\end{aligned}
$$

Now, it is easy to see that $\phi_{q k+1}^{q} \in W_{h}^{p}, 1 \leq k<K$, if and only if $n_{q+1, p+1}^{q ; p}-n_{1,1}^{q ; p}=$ $n_{q+1,1}^{q ; p}=n_{1, p+1}^{q ; p}=0$.

The case $q=p-1$ will be of special interest for mortar finite elements. For simplicity of notation, we denote from now on $N^{p-1 ; p}$ by $N^{p}$ and $n_{i, j}^{p-1 ; p}$ by $n_{i, j}^{p}$. Therefore, we rewrite the general condition (2.3) and obtain that $V_{h}^{p-1} \subset W_{h}^{p}$ if and only if

$$
\begin{aligned}
n_{1,1}^{p} & =n_{p, p+1}^{p} \quad \text { and } \quad n_{p, 1}^{p}=n_{1, p+1}^{p}=0 \\
n_{i, 1}^{p} & =n_{i, p+1}^{p}=0 \text { for all } \quad 2 \leq i \leq p-1 .
\end{aligned}
$$

To get a better feeling, we consider the case $p=2$ in more detail. Then the conditions (2.4) can be simply written as

$$
n_{1,1}^{p}=n_{2,3}^{p} \quad \text { and } \quad n_{2,1}^{p}=n_{1,3}^{p}=0 .
$$

Let us consider $x_{1}^{p}:=-1, x_{2}^{p}:=0$ and $x_{3}^{p}:=1$. Then the basis functions of $\hat{\boldsymbol{\Lambda}}_{p}$ are given by

$$
\begin{aligned}
\lambda_{1}^{p} & =\frac{5}{4} x^{2}-\frac{1}{2} x-\frac{1}{4}, \quad \lambda_{2}^{p}=-\frac{5}{2} x^{2}+\frac{3}{2}, \quad \lambda_{3}^{p}=\frac{5}{4} x^{2}+\frac{1}{2} x+\frac{1}{4}, \\
\lambda_{1}^{p} & =\phi_{1}^{p}-\frac{3}{4} \phi_{2}^{p}+\frac{1}{2}, \quad \lambda_{2}^{p}=\frac{5}{2} \phi_{2}^{p}-1, \quad \lambda_{3}^{p}=\phi_{3}^{p}-\frac{3}{4} \phi_{2}^{p}+\frac{1}{2} ;
\end{aligned}
$$

see [LW02]. Using $\phi_{1}^{p-1}=\phi_{1}^{p}+\frac{1}{2} \phi_{2}^{p}$ and $\phi_{2}^{p-1}=\phi_{3}^{p}+\frac{1}{2} \phi_{2}^{p}$, we find for the entries of the $2 \times 3$ matrix $N^{2}$

$$
\begin{aligned}
& n_{1,1}^{p}=1, \quad n_{1,2}^{p}=\frac{1}{2}, \quad n_{1,3}^{p}=0, \\
& n_{2,1}^{p}=0, \quad n_{2,2}^{p}=\frac{1}{2}, \quad n_{2,3}^{p}=1,
\end{aligned}
$$

and thus (2.5) is satisfied. Unfortunately for $p=3$ and $x_{1}^{p}:=-1, x_{2}^{p}:=-1 / 3$, $x_{3}^{p}:=1 / 3$ and $x_{4}^{p}:=1$, (2.4) is not satisfied. A straightforward calculation shows that the matrix $N^{p}$ for $p=3$ is given by

$$
N^{3}=\left[\begin{array}{cccc}
\frac{11}{15} & \frac{2}{5} & -\frac{1}{5} & 0 \\
\frac{4}{15} & \frac{4}{5} & \frac{4}{5} & \frac{4}{15} \\
0 & -\frac{1}{5} & \frac{2}{5} & \frac{11}{15}
\end{array}\right] .
$$

Although the conditions $n_{1,1}^{3}=n_{3,4}^{3}$ and $n_{3,1}^{3}=n_{1,4}^{3}=0$ are satisfied, we find $n_{2,1}^{3}=n_{2,4}^{3}=\frac{4}{15} \neq 0$. As a result, working with cubic Lagrange finite elements and dual Lagrange multipliers does not yield optimal a priori results for the mortar discretization. To test the performance of the dual Lagrange multiplier space 
TABLE 1. Discretization errors with equidistant nodes (cubic exact solution)

\begin{tabular}{|c|c|c|c|c|c|c|c|}
\hline level & \# elem. & \multicolumn{2}{|c|}{$\left\|u-u_{h}\right\|_{L^{2}(\Omega)}$} & \multicolumn{2}{|c|}{$\left\|u-u_{h}\right\|_{1}$} & \multicolumn{2}{c|}{$\left\|\lambda-\lambda_{h}\right\|_{h}$} \\
\hline 0 & 24 & $2.935203 e-05$ & & $1.970982 e-03$ & & $9.234661 e-02$ & \\
\hline 1 & 96 & $5.862444 e-06$ & 2.32 & $8.170738 e-04$ & 1.27 & $4.589376 e-02$ & 1.01 \\
\hline 2 & 384 & $1.071774 e-06$ & 2.45 & $3.040267 e-04$ & 1.43 & $1.854690 e-02$ & 1.31 \\
\hline 3 & 1536 & $1.916484 e-07$ & 2.48 & $1.097294 e-04$ & 1.47 & $6.966565 e-03$ & 1.41 \\
\hline 4 & 6144 & $3.402984 e-08$ & 2.49 & $3.915526 e-05$ & 1.49 & $2.535323 e-03$ & 1.46 \\
\hline 5 & 24576 & $6.027017 e-09$ & 2.50 & $1.390406 e-05$ & 1.49 & $9.091418 e-04$ & 1.48 \\
\hline 6 & 98304 & $1.067999 e-09$ & 2.50 & $4.926273 e-06$ & 1.50 & $3.236870 e-04$ & 1.49 \\
\hline
\end{tabular}

for cubic mortar finite elements, we solve the Poisson equation $-\Delta u=f$ in the domain $\Omega:=(-1,1) \times(0,1)$ decomposed into two squares and with a nonmatching triangulation. The right-hand side function $f$ and the Dirichlet boundary condition on $\partial \Omega$ are computed by using the exact solution

$$
u(x, y)=x^{3}-3 y^{3}+3 x^{2}+4 x y^{2}-5 y x^{2}+5 y+12 .
$$

Since the given solution is a cubic polynomial, we expect to obtain the exact solution by cubic finite elements and by optimal cubic mortar finite elements. The nonoptimal behavior of cubic mortar finite elements with the dual Lagrange multiplier space can be seen in Table 1. We observe that the convergence rates in the $L^{2}$ and $H^{1}$-norms are only of order 2.5 and 1.5 , respectively, and the convergence rate in the weighted Lagrange multiplier norm is also only of 1.5. The error in the $L^{2}$ and $H^{1}$-norms are measured in the norm as defined in Lemma 3.1. whereas the error in the flux is measured in a weighted $L^{2}$-norm defined by (4.11). Although the finite element space has optimal approximation property, we can only show that the Lagrange multiplier space contains the constant function. Therefore, under the assumption that the error is equally distributed the error in the flux converges with order 1.5; see LW02. The convergence rate of order 1.5 in the $H^{1}$-norm can be explained by using the fact that the error in the $H^{1}$-norm is bounded by the best approximation property of the finite element space and the Lagrange multiplier space; see [LSW05].

In the rest of this section, we construct for general $p$ nodal points $x_{1}^{p}, \ldots, x_{p+1}^{p}$ such that (2.4) holds. To start with, we consider $N^{p}$ in more detail. The Gram matrix of the finite element base $\hat{\mathbf{\Phi}}_{p}$ is given by $M_{p}=G_{\hat{\mathbf{\Phi}}_{p}, \hat{\mathbf{\Phi}}_{p}}$ with the $(i, j)$-th entry $m_{i, j}^{p}=\int_{I} \phi_{i}(\hat{s}) \phi_{j}(\hat{s}) d \hat{s}$. We note that $M_{p} \in \mathbb{R}^{(p+1) \times(p+1)}$ is positive-definite and symmetric. The set of biorthogonal basis functions satisfying (2.1) can be formally given by

$$
\hat{\boldsymbol{\Lambda}}_{p}=D_{p+1} M_{p}^{-1} \hat{\boldsymbol{\Phi}}_{p} \quad \text { with } \quad G_{\hat{\mathbf{\Phi}}, \hat{\mathbf{\Lambda}}}=D_{p+1},
$$

where the entries of the diagonal matrix $D_{p+1}$ are given by $d_{i}^{p} \neq 0$. Moreover, we can find a unique restriction matrix $P_{p}$ such that $\hat{\boldsymbol{\Phi}}_{p-1}=P_{p} \hat{\boldsymbol{\Phi}}_{p}$, yielding

$$
P_{p} M_{p} D_{p+1}^{-1} \hat{\mathbf{\Lambda}}_{p}=\hat{\mathbf{\Phi}}_{p-1},
$$

and thus $N^{p}=P_{p} M_{p} D_{p+1}^{-1}$. We recall that the set $\hat{\boldsymbol{\Phi}}_{p}$ forms a nodal basis associated with the nodes $x_{i}^{p}, 1 \leq i \leq p+1$. Then the entries $P_{i j}^{p}$ of the restriction matrix $P_{p}$ are given by $P_{i j}^{p}=\phi_{i}^{p-1}\left(x_{j}^{p}\right), 1 \leq i \leq p, 1 \leq j \leq p+1$. Using the product form of 
$N^{p}$, we find

$$
n_{i, j}^{p}=\frac{1}{d_{j}^{p}} \sum_{k=1}^{p+1} \phi_{i}^{p-1}\left(x_{k}^{p}\right) \int_{I} \phi_{k}^{p}(\hat{s}) \phi_{j}^{p}(\hat{s}) d \hat{s}=\frac{1}{d_{j}^{p}} \int_{I} \phi_{i}^{p-1}(\hat{s}) \phi_{j}^{p}(\hat{s}) d \hat{s} .
$$

From now on, we assume that the nodal points are symmetric with respect to the origin, i.e.,

$$
x_{i}^{p}=-x_{p+2-i}^{p}, \quad 1 \leq i \leq p+1 .
$$

Lemma 2.3. Let us assume that (A1) holds for $p$ and $p-1$; then

$$
n_{1,1}^{p}=n_{p, p+1}^{p} .
$$

Proof. The assumption (A1) guarantees that $\int_{I} \phi_{i}^{p}(\hat{s}) d \hat{s}=\int_{I} \phi_{p+2-i}^{p}(\hat{s}) d \hat{s}$ and, moreover,

$$
\int_{I} \phi_{1}^{p-1}(\hat{s}) \phi_{1}^{p}(\hat{s}) d \hat{s}=\int_{I} \phi_{p}^{p-1}(\hat{s}) \phi_{p+1}^{p}(\hat{s}) d \hat{s}
$$

Combining (2.4) and Lemma 2.3, we obtain the following corollary.

Corollary 2.4. Under assumption (A1), $V_{h}^{p-1} \subset W_{h}^{p}$ if and only if

$$
\begin{aligned}
\int_{I} \phi_{1}^{p}(\hat{s}) \phi_{i}^{p-1}(\hat{s}) d \hat{s} & =0, & & 2 \leq i \leq p, \\
\int_{I} \phi_{p+1}^{p}(\hat{s}) \phi_{i}^{p-1}(\hat{s}) d \hat{s} & =0, & & 1 \leq i \leq p-1 .
\end{aligned}
$$

We remark that (2.8) is equivalent to the condition that $\phi_{1}^{p}$ and $\phi_{p+1}^{p}$ are in a suitable orthogonal subspace of dimension two, i.e.,

$\phi_{1}^{p} \in \operatorname{span}\left\{\phi_{2}^{p-1}, \ldots, \phi_{p}^{p-1}\right\}^{\perp} \subset \mathcal{P}_{p}(I), \quad \phi_{p+1}^{p} \in \operatorname{span}\left\{\phi_{1}^{p-1}, \ldots, \phi_{p-1}^{p-1}\right\}^{\perp} \subset \mathcal{P}_{p}(I)$.

Observing $x_{1}^{p-1}=x_{1}^{p}$ and $x_{p}^{p-1}=x_{p+1}^{p}$, we find

$$
\phi_{l}^{p}\left(x_{j}^{p}\right) \phi_{i}^{p-1}\left(x_{j}^{p}\right)=0, \quad l=1,2 \leq i \leq p \text { and } l=p+1,1 \leq i \leq p-1 .
$$

Now, we can easily relate condition (2.8) to quadrature formulas. Let us assume for the moment that associated with the symmetric set of nodal points $-1=x_{1}^{p}<$ $\cdots<x_{p+1}^{p}=1$ is a quadrature formula which is exact for all polynomials of degree less than or equal to $2 p-1$. Then

$$
\int_{I} \phi_{l}^{p}(\hat{s}) \phi_{i}^{p-1}(\hat{s}) d \hat{s}=\sum_{j=1}^{p+1} w_{j}^{p} \phi_{l}^{p}\left(x_{j}^{p}\right) \phi_{i}^{p-1}\left(x_{j}^{p}\right)=0
$$

for $l=1,2 \leq i \leq p$ and $l=p+1,1 \leq i \leq p-1$. Here $w_{j}^{p}$ denote the weights of the quadrature formula. It is well known that there exists a unique quadrature formula on $\bar{I}$ with $p+1$ nodes satisfying $x_{1}^{p}=-1$ and $x_{p+1}^{p}=1$, and being exact for all polynomials of degree less than or equal to $2 p-1$. This is the family of Gauß-Lobatto quadrature formulas. The nodes are based on Legendre polynomials and satisfy that the set of nodes is symmetric and all weights $w_{i}^{p}$ are positive. We note that

$$
w_{j}^{p}=\int_{I} \phi_{j}^{p}(\hat{s}) d \hat{s}, \quad 1 \leq j \leq p+1,
$$

and thus we have $d_{j}^{p}=\int_{I} \phi_{j}^{p}(\hat{s}) d \hat{s}$ for all $1 \leq j \leq p+1$. For convenience of the reader, we recall some characteristic properties of the Legendre polynomials $L_{n}(x)$ and of the Gauß-Lobatto points which are relevant to our applications: 
L1. For some $N \in \mathbb{N}$, the set $\mathcal{L}_{N}:=\left\{L_{0}(x), L_{1}(x), \ldots, L_{N}(x)\right\}$ forms an orthogonal system on $(-1,1)$ with respect to the $L^{2}$-inner product.

L2. The Legendre polynomial of degree $n$ has exactly $n$ distinct real zeros in $(-1,1)$.

L3. If $\mathcal{Z}_{n}:=\left\{x_{1}^{n}<x_{2}^{n}<\cdots<x_{n}^{n}\right\}$ is the set of $n$-zeros of the Legendre polynomial $L_{n}(x)$, then the identity $\int_{-1}^{1} f(x) d x=\sum_{k=1}^{n} w_{k}^{n} f\left(x_{k}^{n}\right)$ holds for all $f \in \mathcal{P}_{2 n-1}(-1,1)$ with $w_{k}^{n}=\frac{2}{n L_{n-1}\left(x_{k}^{n}\right) L_{n}^{\prime}\left(x_{k}^{n}\right)}$ for $1 \leq k \leq n$, where $L_{n}^{\prime}(x)=\frac{d L_{n}(x)}{d x}$.

L4. The polynomial $\left(1-x^{2}\right) L_{n}^{\prime}(x)$ is orthogonal to all polynomials of degree less than $n-1$ and has $n+1$-distinct zeros in $[-1,1]$. If $\mathcal{S}_{n}:=\left\{-1=: x_{1}^{n}<\right.$ $\left.x_{2}^{n}<\cdots<x_{n+1}^{n}=: 1\right\}$ is the zeros of polynomial $\left(1-x^{2}\right) L_{n}^{\prime}(x)$, then $\mathcal{S}_{n}$ is the set of Gauß-Lobatto nodes of order $n$.

L5. If $\mathcal{S}_{n}:=\left\{x_{1}^{n}<x_{2}^{n}<\cdots<x_{n+1}^{n}\right\}$ is the set of Gauß-Lobatto nodes of order $n$, then $\int_{-1}^{1} f(x) d x=\sum_{k=1}^{n+1} w_{k}^{n} f\left(x_{k}^{n}\right)$ for all $f \in \mathcal{P}_{2 n-1}(-1,1)$ with $w_{1}^{n}=w_{n+1}^{n}=\frac{2}{n(n+1)}$ and $w_{k}^{n}=\frac{2}{n(n+1)\left(L_{n}\left(x_{k}^{n}\right)\right)^{2}}$ for $2 \leq k \leq n$.

By construction, we specified a set of nodal points such that $V_{h}^{p-1} \subset W_{h}^{p}$. The following theorem shows that this set is unique.

Theorem 2.5. $V_{h}^{p-1} \subset W_{h}^{p}$ if and only if the finite element basis $\hat{\boldsymbol{\Phi}}_{p}$ which defines $W_{h}^{p}$ is based on the Gauß-Lobatto points $\mathcal{S}_{p}:=\left\{-1=: x_{1}^{p}<\cdots<x_{p+1}^{p}:=1\right\}$.

Proof. Let $\mathcal{S}_{p}:=\left\{-1=: x_{1}^{p}<x_{2}^{p}<\cdots<x_{p+1}^{p}:=1\right\}$ be the Gauß-Lobatto points; then the assumption (A1) is satisfied and, moreover, (2.9) holds. Thus, we are in the setting of Corollary 2.4, and we find $V_{h}^{p-1} \subset W_{h}^{p}$. Now, let us assume that we have $\hat{\boldsymbol{\Phi}}_{p}$ such that the associated space $W_{h}^{p}$ satisfies $V_{h}^{p-1} \subset W_{h}^{p}$. We observe that the zeros of the polynomial $(\hat{s}+1) \phi_{1}^{p}(\hat{s})$ are exactly the nodes in $\mathcal{S}_{p}$. In a next step, we show that the polynomial $(\hat{s}+1) \phi_{1}^{p}(\hat{s})$ is orthogonal to the polynomial space $\mathcal{P}_{p-2}(I)$. Let $R(\hat{s})$ be in $\mathcal{P}_{p-2}(I)$. We find that $T(\hat{s}):=(\hat{s}+1) R(\hat{s})$ is in $\mathcal{P}_{p-1}(I)$ and $T(-1)=0$, and thus it can be written as $T(\hat{s})=\sum_{k=2}^{p} T\left(x_{k}^{p}\right) \phi_{k}^{p-1}(\hat{s})$. In terms of (2.4) and (2.7), we find

$$
\int_{I}(\hat{s}+1) \phi_{1}^{p}(\hat{s}) R(\hat{s}) d \hat{s}=\int_{I} \phi_{1}^{p}(\hat{s}) \sum_{k=2}^{p} T\left(x_{k}^{p}\right) \phi_{k}^{p-1}(\hat{s}) d \hat{s}=0 .
$$

The properties of the Legendre polynomials guarantee that the $p+1$-zeros of $(\hat{s}+$ 1) $\phi_{1}^{p}(\hat{s})$ are the same as of $\left(1-\hat{s}^{2}\right) L_{p}^{\prime}(\hat{s})$.

We note that Theorem 2.5 guarantees existence and uniqueness of the set of points. Now, we reconsider the cases $p=2$ and $p=3$. For $p=2$, we find for the Gauß-Lobatto nodes $\mathcal{S}_{2}:=\{-1,0,1\}$, which corresponds to the case of Lagrange finite elements. However, for $p=3$ the Gauß-Lobatto nodes are given by $\mathcal{S}_{3}:=\left\{-1,-\frac{\sqrt{5}}{5}, \frac{\sqrt{5}}{5}, 1\right\}$. This set of nodes is not equidistributed. A simple calculation shows that

$$
N^{3}:=\left[\begin{array}{cccc}
\frac{1}{6} & \frac{1+\sqrt{5}}{12} & \frac{1-\sqrt{5}}{12} & 0 \\
0 & \frac{2}{3} & \frac{2}{3} & 0 \\
0 & \frac{1-\sqrt{5}}{12} & \frac{1+\sqrt{5}}{12} & \frac{1}{6}
\end{array}\right] D_{4}^{-1}=\left[\begin{array}{cccc}
1 & \frac{1+\sqrt{5}}{10} & \frac{1-\sqrt{5}}{10} & 0 \\
0 & \frac{4}{5} & \frac{4}{5} & 0 \\
0 & \frac{1-\sqrt{5}}{10} & \frac{1+\sqrt{5}}{10} & 1
\end{array}\right] .
$$



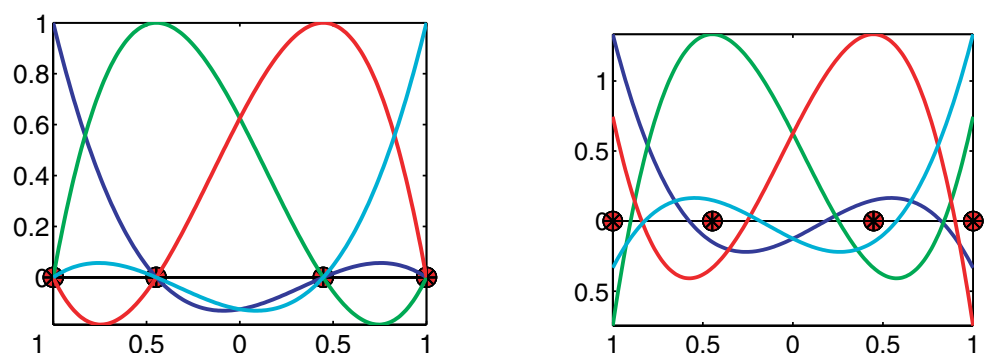

Figure 2.1. The finite element basis functions based on GaußLobatto nodes $\mathcal{S}_{3}$ (left) and the associated biorthogonal basis functions (right).

Having computed $N^{3}$, we can directly verify the conditions (2.4) and find $V_{h}^{p-1} \subset$ $W_{h}^{p}$. Figure 2.1 shows the cubic nodal basis functions on the reference element associated with the Gauß-Lobatto nodes and the corresponding biorthogonal basis functions.

Remark 2.6. Recently, spectral element methods with nodal polynomial interpolation have been of research interest; see, e.g., WPH00, Hes98. Most often GaußLobatto quadrature nodes are used to get a set of interpolation points for the approximation of smooth functions on rectangular meshes. On simplicial meshes such types of nodes are not known. In this case, Fekete points seem to be a natural and good choice; see, e.g., TWV00, PR04. Computational results, TWV00, support the conjecture of Bos Bos91 that Fekete points along the one-dimensional boundary of the triangle are the one-dimensional Gauß-Lobatto points. Additionally, it has been shown in BTW00 that tensor product Gauß-Lobatto points are Fekete points. Hence, working with Fekete points in two-dimensional finite elements one obtains the Gauß-Lobatto nodes along the boundary of triangle or quadrilateral, and the biorthogonal base can easily be formed for the Lagrange multiplier space leading to an optimal scheme for the mortar finite elements in two dimensions.

The next remark is concerned with the quality of convergence of the finite element interpolant based on Gauß-Lobatto and Fekete points.

Remark 2.7. It is well known that working with equally spaced interpolation points, uniform convergence in $p$ of the polynomial interpolation cannot be obtained. The so-called Lebesgue constant $C_{L}(p)$ (see, e.g., TWV00) characterizes the quality of the approximation of the polynomial interpolant. In the case of equally spaced points, $C_{L}(p)$ grows exponentially in $p$. Although the construction of best approximation polynomials are, in general, not known, it can be shown that working with Fekete points reduces the exponential growth of $C_{L}(p)$ to a linear growth TWV00. Moreover, numerical results suggest that $C_{L}(p)$ grows like $\sqrt{p}$ on triangles. In the univariate case, the bound for the Gauß-Lobatto nodes is well known to be logarithmic in $p$ Bru97.

Recently it has been shown that we can find optimal a priori estimates for mortar finite elements if the Lagrange multiplier space has a smaller dimension than the trace space of the finite elements on the slave interface side with zero boundary condition; see [LWW05. However, to get an optimal estimate, the Lagrange 
multiplier space has to reproduce the piecewise polynomial space of degree $p-1$. In the rest of this section, we consider the construction of a biorthogonal basis of smaller dimension. This construction will also be useful for the modification of Lagrange multipliers around some crosspoint considered in the next section. Let $\mathcal{R}_{q}:=\left\{y_{1}<y_{2}<\cdots<y_{q}\right\}$ be a proper subset of $\mathcal{S}_{p}$ consisting of $q$ distinct points of $\mathcal{S}_{p}$ with $q \leq p$, and $\hat{\Phi}_{q}:=\left\{\phi_{l(1)}^{p}, \ldots, \phi_{l(q)}^{p}\right\}, 1 \leq l(k) \leq p+1$, be the subset of $\hat{\mathbf{\Phi}}_{p}$ associated with the nodes in $\mathcal{R}_{q}$, i.e., $x_{l(k)}^{p}=y_{k}, 1 \leq k \leq q$. We note that $\hat{\Phi}_{q}$ spans a $q$-dimensional space and that the set $\hat{\Psi}_{q}:=\left\{\psi_{1}, \ldots, \psi_{q}\right\}$ with the property $\psi_{i} \in \mathcal{P}_{q-1}(I), \psi_{i}\left(y_{j}\right)=\delta_{i j}$ spans the polynomial space of degree $q-1$. The following lemma states the biorthogonality between the elements of $\hat{\Phi}_{q}$ and $\hat{\Psi}_{q}$.

Lemma 2.8. If $\mathcal{S}_{p}$ is the set of $(p+1)$ Gauß-Lobatto nodes in $[-1,1]$, then the basis $\hat{\Phi}_{q}$ is biorthogonal to $\hat{\Psi}_{q}$.

Proof. Using $\phi_{l(k)} \in \mathcal{P}_{p}(I), 1 \leq k \leq q, \psi_{j} \in \mathcal{P}_{q-1}(I), 1 \leq j \leq q$ and $q \leq p$, and applying an exact quadrature formula based on the $(p+1)$ Gauß-Lobatto nodes $\mathcal{S}_{p}$, we find

$$
\begin{aligned}
\int_{I} \phi_{l(k)}(\hat{s}) \psi_{j}(\hat{s}) d \hat{s} & =\sum_{i=1}^{p+1} w_{i}^{p} \phi_{l(k)}\left(x_{i}^{p}\right) \psi_{j}\left(x_{i}^{p}\right)=\sum_{i=1}^{p+1} w_{i}^{p} \delta_{i l(k)} \psi_{j}\left(x_{i}^{p}\right) \\
& =w_{l(k)}^{p} \psi_{j}\left(x_{l(k)}^{p}\right)=w_{l(k)}^{p} \psi_{j}\left(y_{k}\right)=w_{l(k)}^{p} \delta_{j k} .
\end{aligned}
$$

Of special interest for our application are the choice $q=p$ and

$$
\mathcal{R}_{p}^{1}:=\left\{x_{2}^{p}<x_{3}^{p}<\cdots<x_{p+1}^{p}\right\}, \quad \mathcal{R}_{p}^{\mathrm{r}}:=\left\{x_{1}^{p}<x_{2}^{p}<\cdots<x_{p}^{p}\right\} .
$$

The associated biorthogonal sets will be denoted by $\hat{\Psi}_{p}^{1}=\left\{\psi_{1}^{1}, \ldots, \psi_{p}^{1}\right\}$ and $\hat{\Psi}_{p}^{\mathrm{r}}=$ $\left\{\psi_{1}^{\mathrm{r}}, \ldots, \psi_{p}^{\mathrm{r}}\right\}$, respectively.

\section{Application to mortar finite elements}

Here, we briefly recall the mortar finite element method. We consider the following elliptic second order boundary value problem:

$$
\begin{array}{rll}
-\operatorname{div}(a \nabla u)+c u & =f & \text { in } \Omega, \\
u & =0 & \text { on } \partial \Omega,
\end{array}
$$

where $0<a_{0} \leq a \in L^{\infty}(\Omega), f \in L^{2}(\Omega), 0 \leq c \in L^{\infty}(\Omega)$, and $\Omega \subset \mathbb{R}^{2}$, is a bounded polygonal domain. Let $\Omega$ be decomposed into $L$ nonoverlapping polygonal subdomains $\Omega_{l}$, possibly nonconforming, such that $\bar{\Omega}=\bigcup_{l=1}^{L} \bar{\Omega}_{l}$. Each subdomain $\Omega_{l}, 1 \leq l \leq L$, is associated with a simplicial or quadrilateral triangulation $\mathcal{T}_{l ; h_{l}}$ with mesh-size bounded by $h_{l}$. The discrete space of conforming piecewise finite element space of degree $p$ on $\Omega_{l}$ satisfying the homogeneous Dirichlet boundary conditions on $\partial \Omega \cap \partial \Omega_{l}$ is denoted by $X_{l}^{p}$. We assume that we work with nodal finite element basis functions based on Gauß-Lobatto nodes. Then the mortar method is characterized by the introduction of a discrete Lagrange multiplier space $M_{m}^{p}$ on the interfaces $\gamma_{m}, 1 \leq m \leq M$, of the decomposition. For each interface $\gamma_{m}$, there exists a pair $1 \leq r(m)<s(m) \leq L$ such that $\bar{\gamma}_{m}=\partial \Omega_{r(m)} \cap \partial \Omega_{s(m)}$. We assume that $\gamma_{m}$ is a straight line, and we associate with $\gamma_{m}$ a one-dimensional mesh $\mathcal{U}_{m ; h_{m}}$, inherited either from $\mathcal{T}_{r(m) ; h_{r(m)}}$ or from $\mathcal{T}_{s(m) ; h_{s(m)}}$. The subdomain 


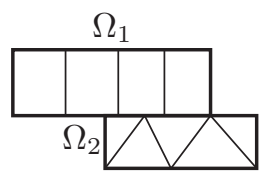

Figure 3.1. A decomposition of $\Omega$ into $\Omega_{1}$ and $\Omega_{2}$ satisfying assumption (3.1) if $\Omega_{2}$ is the slave side.

from which the interface inherits its mesh is called a slave or nonmortar side, and the opposite one a master or mortar side. We do not insist that our decomposition is geometrically conforming. However, to avoid technicalities in the construction of the Lagrange multiplier space, we assume the following:

$$
\left\{\begin{array}{l}
\gamma_{m} \text { is the union of complete one-dimensional faces of } \\
\text { elements on the slave side. }
\end{array}\right.
$$

Note that (3.1) covers special geometrically nonconforming decompositions and is always satisfied for geometrically conforming decompositions. In particular $\gamma_{m}$ does not have to be a full face of $\Omega_{r(m)}$ or $\Omega_{s(m)}$; see Figure 3.1.

To obtain the mortar approximation $u_{h}$, as a solution of a discrete variational problem, there are two main approaches. The first one has been introduced in BMP93, BMP94 and gives rise to a positive definite nonconforming variational problem. It is defined on a subspace $U_{h}^{p}$ of the product space $X_{h}^{p}:=\{v \in$ $\left.L^{2}(\Omega) \mid v_{\left.\right|_{\Omega_{l}}} \in X_{l}^{p}, 1 \leq l \leq L\right\}$. The elements of $U_{h}^{p}$ satisfy a weak continuity condition across the interfaces. The constrained finite element space $U_{h}^{p}$ is given by

$$
U_{h}^{p}:=\left\{v \in X_{h}^{p} \mid \int_{\gamma_{m}}[v] \mu d s=0, \mu \in M_{m}^{p}, 1 \leq m \leq M\right\},
$$

where $[v]$ is the jump of the solution across the interface of the decomposition. Then, the nonconforming formulation of the mortar method can be given in terms of the constrained space $U_{h}^{p}$ : Find $u_{h} \in U_{h}^{p}$ such that

$$
a\left(u_{h}, v_{h}\right)=\left(f, v_{h}\right)_{0}, \quad v_{h} \in U_{h}^{p} .
$$

Here, the bilinear form $a(\cdot, \cdot)$ is defined as

$$
a(v, w):=\sum_{l=1}^{L} \int_{\Omega_{l}} a \nabla v \cdot \nabla w+c v w d x, \quad v, w \in \prod_{l=1}^{L} H^{1}\left(\Omega_{l}\right) .
$$

The second approach is based on equivalent saddle point formulation; see [Ben99]. It is obvious that the quality of the nonconforming approach (3.2) and the properties of $U_{h}^{p}$ depend on the discrete Lagrange multiplier space $M_{h}^{p}:=\prod_{m=1}^{M} M_{m}^{p}$. The optimality of the discretization scheme depends on the verification of the properties of the Lagrange multiplier space presented in LSW05. In the case of dual Lagrange multiplier spaces, it is sufficient to show that $V_{h}^{p-1}\left(\gamma_{m}\right) \subset M_{m}^{p}, 1 \leq m \leq M$. Here $V_{h}^{p-1}\left(\gamma_{m}\right)$ denotes the conforming finite element space of degree $p-1$ associated with the mesh $\mathcal{U}_{m ; h_{m}}$ on $\gamma_{m}$. The error in the Lagrange multiplier $\mu$ is measured in a broken dual norm defined by

$$
\left\|\mu-\mu_{h}\right\|_{M}^{2}=\sum_{m=1}^{L}\left\|\mu-\mu_{h}\right\|_{-1 / 2, \gamma_{m}}^{2},
$$


where $\|\cdot\|_{-1 / 2, \gamma_{m}}$ is the dual norm of $H_{00}^{1 / 2}\left(\gamma_{m}\right)$ and $\mu:=a \frac{\partial u}{\partial n}$.

Lemma 3.1. Under the assumption that the weak solution is smooth enough, we obtain the following a priori estimates for the discretization error:

$$
\frac{1}{h}\left\|u-u_{h}\right\|_{0}+\left\|u-u_{h}\right\|_{1}+\left\|\mu-\mu_{h}\right\|_{M}=\mathcal{O}\left(h^{p}\right)
$$

where $\left\|u-u_{h}\right\|_{s}^{2}:=\sum_{k=1}^{K}\left\|u-u_{h}\right\|_{H^{s}\left(\Omega_{k}\right)}^{2}$ and $h:=\max \left\{h_{1}, \ldots, h_{K}\right\}$.

Unfortunately, we cannot directly apply the results of the previous section to the general mortar situation. The crucial points are the so-called crosspoints. In a geometrical conforming decomposition of the domain of interest in nonoverlapping subdomains, at least three subdomains meet at interior crosspoints, and several interfaces can have crosspoints as a common endpoint. On each interface, we use our newly constructed spaces $W_{h}^{p}$ as a discrete Lagrange multiplier space. Working with the product space of Lagrange multipliers associated with the different interfaces, we obtain too many constraints for the nodal values of the finite element solution at the crosspoints. In other words, the inf-sup condition of the arising saddle point formulation cannot be uniformly satisfied. To obtain a uniform bound for the discretization error, we have to reduce the dimension of the Lagrange multiplier space, and to keep the approach as local as possible we have to reduce the dimension of $W_{h}^{p}$ associated with each interface. Roughly speaking, we have to remove the two degrees of freedom of the Lagrange multiplier space associated with the endpoints of the interfaces. In the rest of this section, we consider the required modifications of the Lagrange multipliers in detail. From now on for simplicity of notation, we skip the index $m$ of the interface $\gamma_{m}$ and call it $\gamma$. Using the same notation as in Section 2, we find that $\boldsymbol{\Phi}_{0 ; p}:=\left\{\phi_{2}^{p}, \ldots, \phi_{p K}^{p}\right\}$ spans the conforming finite element space $V_{0 ; h}^{p}:=V_{h}^{p} \cap H_{0}^{1}(\gamma)$ with zero boundary conditions. Here $K$ denotes the number of edges on the slave side. Now we define a $(p K-1)$-dimensional Lagrange multiplier space by $\boldsymbol{\Lambda}_{0 ; p}:=\left\{\tilde{\lambda}_{2}^{p}, \ldots, \tilde{\lambda}_{p K}^{p}\right\}$, where

$$
\tilde{\lambda}_{j}^{p}:= \begin{cases}\psi_{j-1}^{\mathrm{l}} \circ F_{1}^{-1}, & 2 \leq j \leq p, \\ \psi_{p}^{1} \circ F_{1}^{-1}+\lambda_{2 ; 1}^{p}, & j=p+1, \\ \lambda_{j}^{p}, & p+2 \leq j \leq p(K-1), \\ \psi_{1}^{\mathrm{r}} \circ F_{K}^{-1}+\lambda_{K-1 ; p+1}^{p}, & j=p(K-1)+1, \\ \psi_{j-p(K-1)}^{\mathrm{r}} \circ F_{K}^{-1}, & p(K-1)+2 \leq j \leq p K .\end{cases}
$$

As before, element-wise addition is applied for the sum of two basis functions.

Theorem 3.2. The set $\boldsymbol{\Lambda}_{0 ; p}$ is a dual basis with respect to $\boldsymbol{\Phi}_{0 ; p}$. Moreover $M_{m}^{p}:=$ span $\boldsymbol{\Lambda}_{0 ; p}$ satisfies $V_{h}^{p-1}(\gamma) \subset M_{m}^{p}$.

Proof. By definition of the elements in $\boldsymbol{\Lambda}_{0 ; p}$ and by means of Lemma 2.8, the biorthogonality holds. Following the lines as in the proof of Lemma 2.2, we have to consider the first and the last edge of $\gamma$. We only work out the details for the first edge. Observing $\phi_{j}^{p-1}\left(F_{1}\left(x_{p+1}^{p}\right)\right)=0,1 \leq j \leq p-1$, we find

$$
\phi_{j}^{p-1}=\sum_{i=1}^{p-1} \phi_{j}^{p-1}\left(F_{1}\left(x_{i+1}^{p}\right)\right) \psi_{i}^{1} \circ F_{1}^{-1}=\sum_{i=1}^{p-1} \phi_{j}^{p-1}\left(F_{1}\left(x_{i+1}^{p}\right)\right) \tilde{\lambda}_{i+1}^{p} \in M_{m}^{p} .
$$



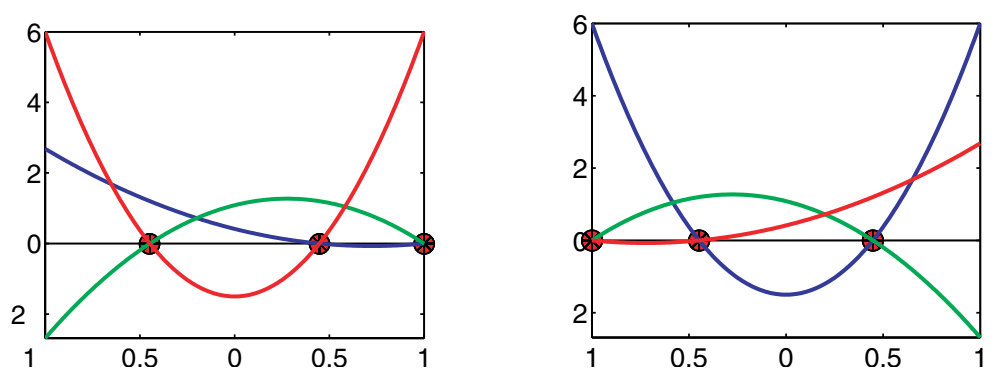

FiguRE 3.2. The modified Lagrange multiplier basis functions at the crosspoints for the cubic case, the first edge (left), and the last edge (right).

A similar consideration for $j=p$ yields that $\phi_{p}^{p-1} \in M_{m}^{p}$ if and only if $n_{1,1}^{p}=1$. Using the explicit form (2.7) of $n_{1,1}^{p}$ and $\phi_{1}^{p-1}(-1)=1$, we find

$$
n_{1,1}^{p}=\frac{1}{d_{1}^{p}} \int_{I} \phi_{1}^{p-1}(\hat{s}) \phi_{1}^{p}(\hat{s}) d \hat{s}=\frac{w_{1}^{p}}{\int_{I} \phi_{1}^{p}(\hat{s}) d \hat{s}}=1 .
$$

We have shown the modified Lagrange multiplier basis functions in the case of the first and the last edge in Figure 3.2

Using this type of Lagrange multiplier space in the definition of a mortar finite element method, we obtain optimal a priori estimates, and the mass matrix on the slave side is a diagonal one. Now, we briefly discuss the extension of our onedimensional construction of dual Lagrange multiplier space to the two-dimensional case in a reference element $T$, where $T$ is a square $(-1,1) \times(-1,1)$ or a triangle $\{(x, y), x \geq 0, y \geq 0, x+y \leq 1\}$ depending on the quadrangulation or triangulation of the domain. The following remark is concerned with the extension to the two-dimensional case. Unfortunately, in the case of triangle, the straightforward construction as in the case of a quadrilateral is not possible.

Remark 3.3. Working with a tensor product finite element space from one dimension in quadrilateral mesh, we can apply the idea of one-dimensional construction in a straightforward way. However, since Gauß-Lobatto nodes are not known for the triangle, the situation with simplicial mesh is much more complicated. The Fekete points in a triangle do not show the nice property of Gauß-Lobatto nodes that any polynomial of degree $2 p-1$ can be integrated exactly with a suitable choice of weights. Hence one can easily show that a basis biorthogonal to a nodal finite element base does not exist for a triangle with optimal approximation property for $p \geq 2$. In this case, one can look for a basis biorthogonal to a proper subset of finite element space $\hat{\boldsymbol{\Phi}}_{p}$ as described above or a basis $\hat{\boldsymbol{\Lambda}}_{p}$ which leads to a lower or upper triangular mass matrix. We refer to LSW05 for the quadratic and cubic case. Although there does not exist a straightforward relation between a quadrature formula and a biorthogonal base in a triangle as in quadrilaterals, from the theory presented above one can figure out that there exists some form of relationship even in this case. 


\section{Numerical Results}

In this section, we present numerical results illustrating the performance of cubic mortar finite elements based on our new dual Lagrange multiplier space. In particular, we present the discretization errors in the $L^{2}$ - and $H^{1}$-norm for the primal variable, and a weighted Lagrange multiplier norm defined by

$$
\left\|\lambda-\lambda_{h}\right\|_{h}^{2}:=\sum_{m=1}^{M} \sum_{e \in \mathcal{U}_{m ; h_{m}}} h_{e}\left\|\lambda-\lambda_{h}\right\|_{0 ; e}^{2},
$$

where $h_{e}$ is the length of the edge $e$ on the slave side. Numerical results on linear and quadratic mortar finite elements with dual Lagrange multiplier spaces can be found in Woh01, LW02]. The flexibility of mortar methods allows us to consider geometrically nonconforming decompositions, nonconvex subdomains, and crosspoints. Starting with a conforming coarse triangulation on each subdomain, we apply uniform refinement in each step.

In our first example, we consider a problem with many crosspoints. Here, we consider $-\Delta u=f$ in $\Omega$, where $\Omega$ is the unit square $(0,1)^{2}$. The domain $\Omega$ is decomposed into nine squares with $\Omega_{i j}:=((i-1) / 3, i / 3) \times((j-1) / 3, j / 3), 1 \leq$ $i \leq 3,1 \leq j \leq 3$. The right-hand side $f$ and the Dirichlet boundary conditions are chosen such that the exact solution is given by

$$
u(x, y)=\sin (3.5 \pi y+x) e^{y+(1-x)^{2}}+\cos (3.5 \pi x+y) e^{x+(1-y)^{2}} .
$$

In Figure 4.1, the decomposition of the domain, the initial finite element partition, and the isolines of the solution are shown. Here, we have four interior crosspoints and 12 interfaces, and the master and slave sides are chosen randomly. The discretization errors versus the number of elements along with the order of convergence at each refinement step are given in Table 2, We observe the optimal asymptotic
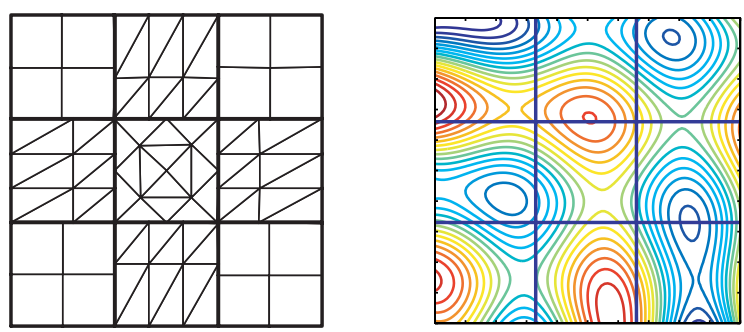

Figure 4.1. Decomposition of the domain and initial partitions (left) and isolines of the solution (right) for Example 1.

TABLE 2. Discretization errors for Example 1

\begin{tabular}{|c|c|c|c|c|c|c|c|}
\hline level & $\#$ elem. & \multicolumn{2}{|c|}{$\left\|u-u_{h}\right\|_{L^{2}(\Omega)}$} & \multicolumn{2}{|c|}{$\left\|u-u_{h}\right\|_{1}$} & \multicolumn{2}{c|}{$\left\|\lambda-\lambda_{h}\right\|_{h}$} \\
\hline 0 & 80 & $9.428500 e-04$ & & $8.682827 e-03$ & & $9.036892 e-03$ & \\
\hline 1 & 320 & $5.753638 e-05$ & 4.03 & $1.039127 e-03$ & 3.06 & $5.542193 e-04$ & 4.03 \\
\hline 2 & 1280 & $3.539360 e-06$ & 4.02 & $1.268866 e-04$ & 3.03 & $5.572755 e-05$ & 3.31 \\
\hline 3 & 5120 & $2.208067 e-07$ & 4.00 & $1.575260 e-05$ & 3.01 & $6.857704 e-06$ & 3.02 \\
\hline 4 & 20480 & $1.375295 e-08$ & 4.00 & $1.960257 e-06$ & 3.01 & $5.880097 e-07$ & 3.54 \\
\hline 5 & 81920 & $8.586956 e-10$ & 4.00 & $2.445284 e-07$ & 3.00 & $5.020655 e-08$ & 3.55 \\
\hline
\end{tabular}



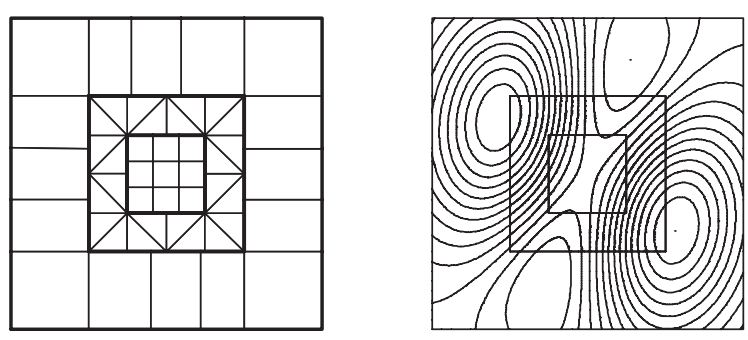

Figure 4.2. Decomposition of the domain and initial partitions (left) and isolines of the solution (right) for Example 2.

TABLE 3. Discretization errors for Example 2

\begin{tabular}{|c|c|c|c|c|c|c|c|}
\hline level & \# elem. & \multicolumn{2}{|c|}{$\left\|u-u_{h}\right\|_{L^{2}(\Omega)}$} & \multicolumn{2}{|c|}{$\left\|u-u_{h}\right\|_{1}$} & \multicolumn{2}{|c|}{$\left\|\lambda-\lambda_{h}\right\|_{h}$} \\
\hline 0 & 49 & $1.709480 e-03$ & & $1.323415 e-02$ & & $1.293947 e-03$ & \\
\hline 1 & 196 & $1.533203 e-04$ & 3.48 & $2.584120 e-03$ & 2.36 & $7.148655 e-05$ & 4.18 \\
\hline 2 & 784 & $9.767404 e-06$ & 3.97 & $3.289095 e-04$ & 2.97 & $9.127343 e-06$ & 2.97 \\
\hline 3 & 3136 & $6.127065 e-07$ & 3.99 & $4.124910 e-05$ & 3.00 & $7.273459 e-07$ & 3.65 \\
\hline 4 & 12544 & $3.833414 e-08$ & 4.00 & $5.160192 e-06$ & 3.00 & $6.608121 e-08$ & 3.46 \\
\hline 5 & 50176 & $2.396388 e-09$ & 4.00 & $6.451090 e-07$ & 3.00 & $5.678886 e-09$ & 3.54 \\
\hline
\end{tabular}

rates of convergence both in the $L^{2}$ - and $H^{1}$-norms. We find that the convergence in the energy error is of order $h^{3}$, whereas the convergence of the error in the $L^{2}$ norm is of order $h^{4}$. The rate of convergence in the weighted Lagrange multiplier norm is of order $\frac{7}{2}$, which is the same order as the best approximation.

In our second example, we consider the decomposition of the unit square into three subdomains with two nonconvex subdomains. The middle subdomain is chosen as the slave side. We have shown the decomposition of the domain and initial nonmatching triangulation in the left picture of Figure 4.2, and the isolines of the solution are shown in the right. The right-hand side $f$ and the Dirichlet boundary conditions of $-\Delta u=f$ are chosen from the exact solution, which is given by

$$
u(x, y)=x(x-y) e^{-10.0(x-0.5)^{2}-6.0(y-0.5)^{2}} .
$$

The discretization errors in the $L^{2}-, H^{1}-$, and in the weighted Lagrange multiplier norm are presented in Table 3. Although two subdomains are nonconvex, we observe optimal convergence rates for all considered norms.

In our third example, we decompose the unit square in four subdomains defined by

$$
\Omega_{i j}:=((i-1) / 2, i / 2) \times((j-1) / 2, j / 2), 1 \leq i \leq 2,1 \leq j \leq 2 .
$$

In this example, the problem is defined by

$$
-\operatorname{div}(a \nabla u)+u=f \quad \text { in } \Omega,
$$

where $a$ is chosen to be one in $\Omega_{11}$ and $\Omega_{22}$, and three in $\Omega_{21}$ and $\Omega_{12}$. Figure 4.3 shows the decomposition into four subdomains, our initial nonmatching triangulation and the isolines of the solution. Here, we choose the exact solution $u(x, y)=(x-1 / 2)(y-1 / 2) \exp \left(-10(x-1 / 2)^{2}-5(y-1 / 2)^{2}\right) / a$; see Figure 4.3. We point out that the normal derivative of the solution across the interface has a jump, but the flux is continuous. We have given the discretization errors in Table 4 As 

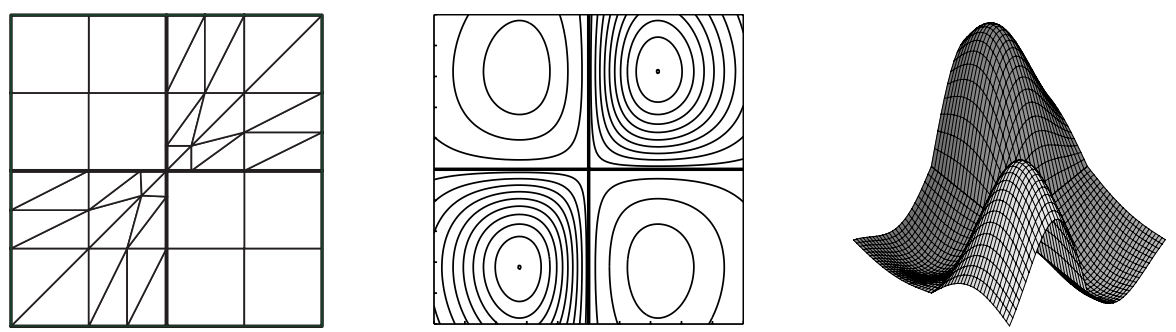

Figure 4.3. Decomposition of the domain and initial partitions (left), isolines of the solution (middle), and exact solution (right) for Example 3.

TABlE 4. Discretization errors for Example 3

\begin{tabular}{|c|c|c|c|c|c|c|c|}
\hline level & \# elem. & \multicolumn{2}{|c|}{$\left\|u-u_{h}\right\|_{L^{2}(\Omega)}$} & \multicolumn{2}{|c|}{$\left\|u-u_{h}\right\|_{1}$} & \multicolumn{2}{c|}{$\left\|\lambda-\lambda_{h}\right\|_{h}$} \\
\hline 0 & 44 & $3.096840 e-03$ & & $1.532198 e-02$ & & $1.954521 e-03$ & \\
\hline 1 & 176 & $2.053727 e-04$ & 3.91 & $1.877596 e-03$ & 3.03 & $1.396639 e-04$ & 3.81 \\
\hline 2 & 704 & $1.290557 e-05$ & 3.99 & $2.348717 e-04$ & 3.00 & $1.025518 e-05$ & 3.77 \\
\hline 3 & 2816 & $7.940191 e-07$ & 4.02 & $2.917781 e-05$ & 3.01 & $8.292942 e-07$ & 3.63 \\
\hline 4 & 11264 & $4.913603 e-08$ & 4.01 & $3.636055 e-06$ & 3.00 & $7.165627 e-08$ & 3.53 \\
\hline 5 & 45056 & $3.055406 e-09$ & 4.01 & $4.538339 e-07$ & 3.00 & $6.169664 e-09$ & 3.54 \\
\hline
\end{tabular}

in the previous two examples, we observe the optimal asymptotic convergence rates in the $L^{2}$ and $H^{1}$-norms and a better convergence rate in the weighted Lagrange multiplier norm.

In our last example, we decompose the domain, shown in Figure 4.4, into three subdomains in a geometrically nonconforming way satisfying assumption (3.1). The subdomains are given by $\Omega_{1}:=(1.5,2.5) \times(0,1), \Omega_{2}:=(0,4) \times(1,2)$, and $\Omega_{3}:=$ $(1.5,2.5) \times(2,3)$, where the slave sides are set to be on $\Omega_{1}$ and $\Omega_{2}$. Our last problem is defined by

$$
\begin{aligned}
-\Delta u & =f \text { in } \Omega \text { with } \\
\left.\frac{\partial u}{\partial \mathbf{n}}\right|_{\Gamma_{N}} & =0, \quad \text { and }\left.u\right|_{\Gamma_{D}}=g
\end{aligned}
$$

where

$$
\begin{gathered}
\Gamma_{N}=\left\{(x, 1) \in \mathbb{R}^{2}: 0<x<1.5\right\} \cup\left\{(x, 1) \in \mathbb{R}^{2}: 2.5<x<4\right\} \\
\cup\left\{(x, 2) \in \mathbb{R}^{2}: 0<x<1.5\right\} \cup\left\{(x, 2) \in \mathbb{R}^{2}: 2.5<x<4\right\}
\end{gathered}
$$

and

$$
\Gamma_{D}:=\partial \Omega \backslash \Gamma_{N} .
$$

The right-hand side and the function $g$ of the problem are determined from the exact solution

$$
u(x, y)=e^{-1.5(y-1.5)^{2}} x(x-4) y(y-3)(y-1)^{2}(y-2)^{2}(\sin (5 x)+\cos (5 y)),
$$

which satisfies the homogeneous natural boundary condition on $\Gamma_{N}$. The isolines of the solution are given in the right picture of Figure 4.4, and the discretization errors in the $\mathrm{L}^{2}$-, $\mathrm{H}^{1}$-, and the weighted Lagrange multiplier norm are given in Table 5. Having a geometrically nonconforming decomposition does not affect the optimality of the method. 

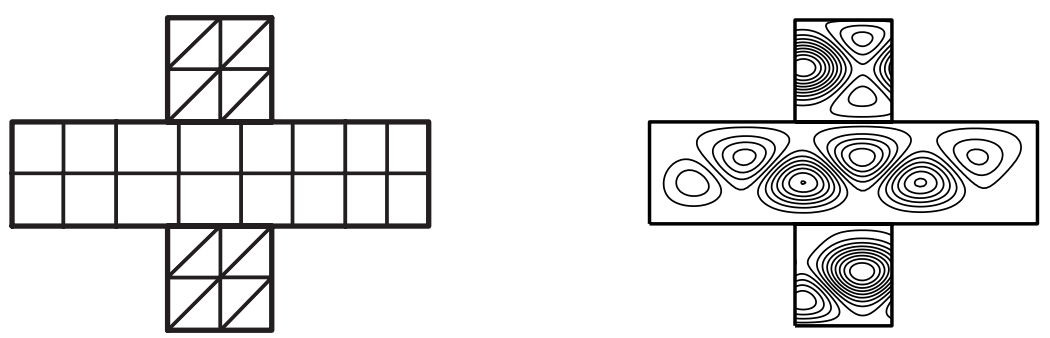

Figure 4.4. Decomposition of the domain and initial partitions (left) and isolines of the solution (right) for Example 4.

TABLE 5. Discretization errors for Example 4

\begin{tabular}{|c|c|c|c|c|c|c|c|}
\hline level & \# elem. & \multicolumn{2}{|c|}{$\left\|u-u_{h}\right\|_{L^{2}(\Omega)}$} & \multicolumn{2}{|c|}{$\left\|u-u_{h}\right\|_{1}$} & \multicolumn{2}{c|}{$\left\|\lambda-\lambda_{h}\right\|_{h}$} \\
\hline 0 & 32 & $6.514124 e-02$ & & $1.547446 e-01$ & & $2.777673 e-01$ & \\
\hline 1 & 128 & $5.454630 e-03$ & 3.58 & $3.295539 e-02$ & 2.23 & $4.888302 e-02$ & 2.51 \\
\hline 2 & 512 & $3.409338 e-04$ & 4.00 & $3.839702 e-03$ & 3.10 & $2.402761 e-03$ & 4.35 \\
\hline 3 & 2048 & $2.117516 e-05$ & 4.01 & $4.820277 e-04$ & 2.99 & $1.647853 e-04$ & 3.87 \\
\hline 4 & 8192 & $1.317693 e-06$ & 4.01 & $6.030857 e-05$ & 3.00 & $1.459654 e-05$ & 3.50 \\
\hline 5 & 32768 & $8.217674 e-08$ & 4.00 & $7.539048 e-06$ & 3.00 & $1.362018 e-06$ & 3.42 \\
\hline 6 & 131072 & $5.137190 e-09$ & 4.00 & $9.423127 e-07$ & 3.00 & $1.198391 e-07$ & 3.51 \\
\hline
\end{tabular}

\section{REFERENCES}

[Ben99] F. Ben Belgacem. The mortar finite element method with Lagrange multipliers. Numer. Math., 84:173-197, 1999.

[Ben04] F. Ben Belgacem. A stabilized domain decomposition method with nonmatching grids for the stokes problem in three dimensions. SIAM J. Numer. Anal., 42:667-685, 2004.

[BMP93] C. Bernardi, Y. Maday, and A.T. Patera. Domain decomposition by the mortar element method. In H. Kaper et al., editor, Asymptotic and numerical methods for partial differential equations with critical parameters, pages 269-286. Reidel, Dordrecht, 1993.

[BMP94] C. Bernardi, Y. Maday, and A.T. Patera. A new nonconforming approach to domain decomposition: the mortar element method. In H. Brezzi et al., editor, Nonlinear partial differential equations and their applications, pages 13-51. Paris, 1994.

[Bos91] L. Bos. On certain configurations of points in $\mathbb{R}^{n}$ which are unisolvent for polynomial interpolation. J. Approx. Theory, 64:271-280, 1991.

[Bru97] L. Brutman. Lebesgue functions for polynomial interpolation-a survey. Ann. Numer. Math., 4:111-127, 1997.

[BTW00] L. Bos, M.A. Taylor, and B.A. Wingate. Tensor product Gauss-Lobatto points are Fekete points for the cube. Math. Comp., 70:1543-1547, 2000.

[DS99] W. Dahmen and R.P. Stevenson. Element-by-element construction of wavelets satisfying stability and moment conditions. SIAM J. Numer. Anal., 37(1):319-352, 1999.

[Hes98] J. S. Hesthaven. From electrostatics to almost optimal nodal sets for polynomial interpolation in a simplex. SIAM J. Numer. Anal., 35:655-676, 1998.

[HT04a] P. Hauret and P. Le Tallec. Stabilized discontinuous mortar formulation for elastostatics and elastodynamics problems - Part I: formulation and analysis. CMAP Internal Report 553, 2004.

[HT04b] P. Hauret and P. Le Tallec. A stabilized discontinuous mortar formulation for elastostatics and elastodynamics problems - Part II: discontinuous Lagrange multipliers. CMAP Internal Report 554, 2004. 
[KLPV01] C. Kim, R.D. Lazarov, J.E. Pasciak, and P.S. Vassilevski. Multiplier spaces for the mortar finite element method in three dimensions. SIAM J. Numer. Anal., 39:519538,2001

[LSW05] B.P. Lamichhane, R.P. Stevenson, and B.I. Wohlmuth. Higher order mortar finite element methods in 3D with dual Lagrange multiplier bases. Numer. Math., 102:93121, 2005.

[LW02] B.P. Lamichhane and B.I. Wohlmuth. Higher order dual Lagrange multiplier spaces for mortar finite element discretizations. CALCOLO, 39:219-237, 2002.

[Mar05] L. Marcinkowski. A mortar finite element method for fourth order problems in two dimensions with Lagrange multipliers. SIAM J. Numer. Anal., 42:1998-2019, 2005.

[OW01] P. Oswald and B.I. Wohlmuth. On polynomial reproduction of dual FE bases. In N. Debit, M. Garbey, R. Hoppe, J. Pèriaux, D. Keyes, and Y. Kuznetsov, editors, Thirteenth International Conference on Domain Decomposition Methods, pages 8596, 2001.

[PR04] R. Pasquetti and F. Rapetti. Spectral element methods on triangles and quadrilaterals: comparisons and applications. J. Comp. Phy., 198:349-362, 2004.

[Ste03] R. Stevenson. Locally supported, piecewise polynomial biorthogonal wavelets on nonuniform meshes. Constr. Approx., 19:477-508, 2003.

[TWV00] M.A. Taylor, B.A. Wingate, and R.E. Vincent. An algorithm for computing Fekete points in the triangle. SIAM J. Numer. Anal., 38:1707-1720, 2000.

[Woh00] B.I. Wohlmuth. A mortar finite element method using dual spaces for the Lagrange multiplier. SIAM J. Numer. Anal., 38:989-1012, 2000.

[Woh01] B.I. Wohlmuth. Discretization Methods and Iterative Solvers Based on Domain Decomposition, volume 17 of LNCS. Springer Heidelberg, 2001.

[WPH00] T. Warburton, L.F. Pavarino, and J.S. Hesthaven. A pseudo-spectral scheme for the incompressible Navier-Stokes equations using unstructured nodal elements. J. Comp. Phy., 164:1-21, 2000.

Institute of Applied Analysis and Numerical Simulation, University of Stuttgart, Stuttgart, Germany

E-mail address: lamichhane@mathematik.uni-stuttgart.de

Institute of Applied Analysis and Numerical Simulation, University of Stuttgart, Stuttgart, Germany

E-mail address: wohlmuth@mathematik.uni-stuttgart.de 